\title{
Lettre à la rédaction : Suicide par ingestion massive d'acébutolol
}

\section{Acebutolol fatal self-poisoning : a case report}

\section{Introduction}

L'acébutolol $\left(\mathrm{C}_{18} \mathrm{H}_{28} \mathrm{~N}_{2} \mathrm{O}_{4}\right)$ est un $\beta$-bloquant adrénergique utilisé pour le traitement de l'hypertension puisqu'il renforce, régularise et ralenti le muscle cardiaque. Il est commercialisé sous la dénomination Sectral ${ }^{\circledR}$.

Les concentrations thérapeutiques dans le sang sont comprises entre 0,3 et $1,3 \mathrm{mg} / \mathrm{L}$, pour une posologie journalière habituelle de $400 \mathrm{mg}$ de Sectral $^{\circledR}$ (1).

En se métabolisant, l'acébutolol va entrainer la formation de deux molécules, un métabolite I (desamide-acébutolol) et du diacétolol, ce dernier ayant des effets pharmacologiques similaires à ceux de l'acébutolol.

L'élimination est principalement urinaire et quasi-totale en 4 jours (1).

Un surdosage en acébutolol peut entrainer une bradycardie, une hypotension, un collapsus et la mort par défaillance cardiovasculaire.

Les décès, suite à l'ingestion massive d'acébutolol sont rares (1), mais récemment, nous avons été amenés à développer une méthode de détection et de quantification de l'acébutolol par chromatographie en phase liquide couplée à la spectrométrie de masse (CLHP/SM) dans le cadre d'un suicide.

\section{Cas médico-légal}

En août 2002, la brigade de recherche de Saint-Diédes-Vosges était chargée de déposer, à l'Institut médico-légal de Strasbourg, un échantillon de sang d'un homme de 46 ans retrouvé mort dans une grotte, après avoir eu une altercation avec sa femme.

Le prélèvement de sang avait été réalisé lors de la levée de corps. Aucun autre renseignement ne nous a été fourni.

\section{Matériels et méthodes}

\section{Réactifs}

Le chloroforme, le méthanol et l'acétonitrile sont de 
qualité HPLC (Merk, Allemagne). L'isopropanol (Prolabo, France) et le $n$-heptane ( Carlo Erba, France) sont de qualité analytique.

Le formate d'ammonium provient de chez Biochemika (France, pureté à $99 \%$ ) et l'acide formique de Prolabo (ultra pur 99-100\%).

L'acébutolol provient de chez Sigma (France) et le cartéolol nous a été généreusement donné par les laboratoires Chauvin (Montpellier, France).

Le chlorure d'ammonium provient de Riedel-de Haën (France) et l'ammoniaque à $28 \%$ de Prolabo.

\section{Préparation des tampons}

Le tampon de la phase mobile est préparé en pesant $63,1 \mathrm{mg}$ de formate d'ammonium dissout dans $490 \mathrm{~mL}$ d'eau distillée. Cette solution est ajusté à pH 3,0 avec de l'acide formique, puis complété à $500 \mathrm{~mL}$.

Le tampon d'extraction est une solution saturée de $\mathrm{NH}_{4} \mathrm{Cl}(25-28 \% \mathrm{v} / \mathrm{v})$, diluée au 1/4 par de l'eau déionisée et ajustée à $\mathrm{pH} 9,5$ par ajout d'ammoniaque concentré.

La solution de cartéolol a été préparée à $100 \mathrm{mg} / \mathrm{L}$ et l'acébutolol aux concentrations de 100 et $10 \mathrm{mg} / \mathrm{L}$, dans du méthanol.

\section{Analyses toxicologiques}

Le dosage de la carboxyhémoglobine a été effectué sur CO-oxymètre (Radiometers Copenhagen, OSM3).

L'alcoolémie a été déterminée par chromatographie en phase gazeuse avec détection par ionisation de flamme (CPG/FID).

Une première recherche qualitative de médicaments a été effectuée par immuno-analyse en utilisant des réactifs EMIT ou FPIA. La recherche de stupéfiants (opiacés, cocaïne, cannabis et amphétamines) a été réalisée par technique ELISA (Cozart).

Dans un second temps, cette recherche a été complétée par une analyse par chromatographie liquide haute performance couplée à une barrette de diodes (CLHP/BD) après extraction liquide/liquide de $1 \mathrm{~mL}$ de sang avec 5 $\mathrm{mL} \mathrm{du}$ mélange chloroforme/isopropanol $/ n$-heptane $(25: 10: 65 ; \mathrm{v} / \mathrm{v})$ à $\mathrm{pH} 9,5$ en présence de prazépam (étalon interne : EI).

Pour la confirmation par CLHP/SM, le sang a été réextrait suivant le même procédé mais en additionnant le cartéolol comme étalon interne.

\section{Instrumentation}

Le système utilisé est composé d'une pompe Applied Biosystems (140B Solvent Delivery System), d'un injecteur automatique Perkin Elmer (séries 200 autosampler) équipé avec une boucle de $5 \mu \mathrm{L}$ et d'un spectromètre de masse PE SCIEX (modèle API 100) avec une interface de type electrospray. La colonne chromatographique est une C18 (LC Packings) de $15 \mathrm{~cm}$ de long et de $1 \mathrm{~mm}$ de diamètre interne $(5 \mu \mathrm{m}$ de diamètre des particules). Le débit de la phase mobile (acétonitrile et tampon $\mathrm{NH}_{4} \mathrm{COOH}$ à $2 \mathrm{mmol} / \mathrm{L} \mathrm{pH} 3,0$ ) dans la colonne est de $50 \mu \mathrm{L} / \mathrm{min}$, avec un gradient d'acétonitrile de $40 \%$ à $90 \%$ en 9 min, plateau à $90 \%$ pendant 6 min et retour à $40 \%$ à la $15^{\text {eme }} \min$.

L'acquisition a été réalisée en mode spectre complet pour une gamme de masses allant de 100 à 450 uma.

Le spectromètre de masse a été optimisé pour les paramètres suivant : électrospray : $4100 \mathrm{~V}$; tension d'orifice : $35 \mathrm{~V}$; anneau de focalisation : $275 \mathrm{~V}$; Q0 : $-8 \mathrm{~V}$.

\section{Paramètres de validation}

La courbe de calibration a été obtenue après extraction, par le procédé établi, de $1 \mathrm{~mL}$ de sang total témoin (négatif en B-bloquant) additionné de cartéolol (EI) à concentration finale de $1 \mathrm{mg} / \mathrm{L}$ et d'acébutolol aux concentrations finales de 0,$1 ; 0,5 ; 1,0 ; 2,5 ; 5,0$ et 10,0 $\mathrm{mg} / \mathrm{L}$.

Pour la répétabilité, dix échantillons additionnés d'acébutolol à la concentration finale de $1 \mathrm{mg} / \mathrm{L}$, ont été extrait, le même jour, par le procédé décrit.

Le rendement d'extraction a été calculé, pour une concentration de $5 \mathrm{mg} / \mathrm{L}$ en acébutolol, en faisant le rapport pour une même quantité d'acébutolol, soit injecté directement, soit extrait par le procédé décrit.

\section{Résultats}

\section{Recherche à large spectre}

La recherche de stupéfiants et de médicaments dans le sang s'est révélée négative, par les techniques d'immuno-détection.

La carboxyhémoglobine dans l'échantillon était inférieure à $1 \%$.

L'alcoolémie est positive avec une concentration à $1,03 \mathrm{~g} / \mathrm{L}$.

La recherche à large spectre par CLHP/BD a permis de détecter un B-bloquant identifié comme étant de l'acébutolol.

Dans le cadre d'affaires médico-légales, il est recommandé de confirmer tout résultat positif par une seconde méthode indépendante de la première. C'est la raison pour laquelle nous avons mis au point une détermination par CLHP/SM.

\section{Confirmation par CLHP/SM}

\section{Validation}

Dans les conditions opératoires décrites, il n'a pas été noté une quelconque interférence des constituants du 
sang avec la substance recherchée ou l'EI.

Dans les conditions chromatographiques et les paramètres de détection définis, les temps de rétentions et les ions de l'acébutolol et de l'EI sont ceux rapportés dans le tableau I.

Les résultats concernant les paramètres de validation sont donnés dans le tableau II. La réponse du détecteur est linéaire pour des concentrations en acébutolol variant de 0,1 à $10,0 \mathrm{mg} / \mathrm{L}$ avec un coefficient de corrélation $R^{2}=0,993$ et une droite d'équation $y=1,197 x$ $+0,2398$. Le coefficient de variation (répétabilité intrajour à $1 \mathrm{mg} / \mathrm{L}$ ) est de $11,8 \%$ et un rendement d'extraction de $60,2 \%$. La limite de détection, calculé pour un $\mathrm{S} / \mathrm{N}=3$, est de $0,03 \mathrm{mg} / \mathrm{L}$. La limite de quantification correspond au point le plus bas de la gamme de linéarité, soit $0,1 \mathrm{mg} / \mathrm{L}$.

La sensibilité de la méthode mise en place apparait suffisante pour la détection de concentrations toxiques et thérapeutiques d'acébutolol.

Tableau I : Temps de rétention (Tr) et fragments ioniques $(\mathrm{m} / \mathrm{z})$ des différentes molécules recherchées.

\begin{tabular}{|c|c|c|}
\hline & Acébutolol & Cartéolol \\
\hline $\operatorname{Tr}(\mathrm{min})$ & 7,13 & 5,45 \\
\hline $\mathrm{m} / \mathrm{z}$ & $\underline{337} ; 319 ; 260$ & $\underline{293} ; 237 ; 202$ \\
\hline
\end{tabular}

les ions soulignés ont été utilisés pour la quantification.

Tableau II : Paramètres de validation de la méthode développée pour la détection de l'acébutolol par CLHP/SM.

\begin{tabular}{|c|c|}
\hline Linéarité $(0,1$ à $10,0 \mathrm{mg} / \mathrm{L})$ & $\mathrm{y}=1,197 \mathrm{x}+0,2398 \quad \mathrm{R}^{2}=0,993$ \\
\hline Rendement d'extraction & $60,2 \%$ \\
\hline Coefficient de variation $(1 \mathrm{mg} / \mathrm{L})$ & $11,8 \%$ \\
\hline Limite de détection $(\mathrm{S} / \mathrm{N}=3)$ & $0,03 \mathrm{mg} / \mathrm{L}$ \\
\hline Limite de quantification & $0,10 \mathrm{mg} / \mathrm{L}$ \\
\hline
\end{tabular}

\section{Cas judiciaire}

Au temps de rétention de 7,13 min (figure 1), le spectre spécifique de l'acébutolol a pu être identifié.

La concentration mesurée était de 37,0 mg/L. Elle se situe dans l'intervalle classique des concentrations mortelles, retrouvée dans la littérature, c'est à dire de 22 à $149 \mathrm{mg} / \mathrm{L}$.

Les décès par acébutolol sont assez rares. Seule une dizaine de cas (2) (3) ont été recencés entre 1979 et 1992. Plus récemment, 2 autres cas ont été rapportés (4) et les auteurs précisaient que l'acébutolol apparait comme le plus toxique des B-bloquants.

\section{Conclusion}

Nous avons développé une technique sensible et spécifique, par CLHP/SM, pouvant mettre en évidence la présence d'acébutolol, dans un échantillon sanguin, que ce soit à des concentrations thérapeutiques ou toxiques.

\section{Références}

1. Randall C. Baselt. Acebutolol. Disposition of toxic drugs and chemicals in man (Fifth edition) $2000: 1-2$.

2. A. Tracqui, P. Kintz, P. Wendling, S. Ritter-Lohner, P. Mangin, A. Jaeger. Toxicological findings in a fatal case of acebutolol self-poisoning. J. Anal. Toxicol. $1992 ; 16$ : $398-400$

3. J.M. Chennebault, A.Turcont, P. Harry, P. Alquier, P. Allain. Fatal poisonning by acebutolol. Therapie 1986 ; $41: 143$.

4. J.N. Love. Acebutolol overdose resulting in fatalities. $J$. Emerg. Med. 2002 ; 18 : 341-4. 


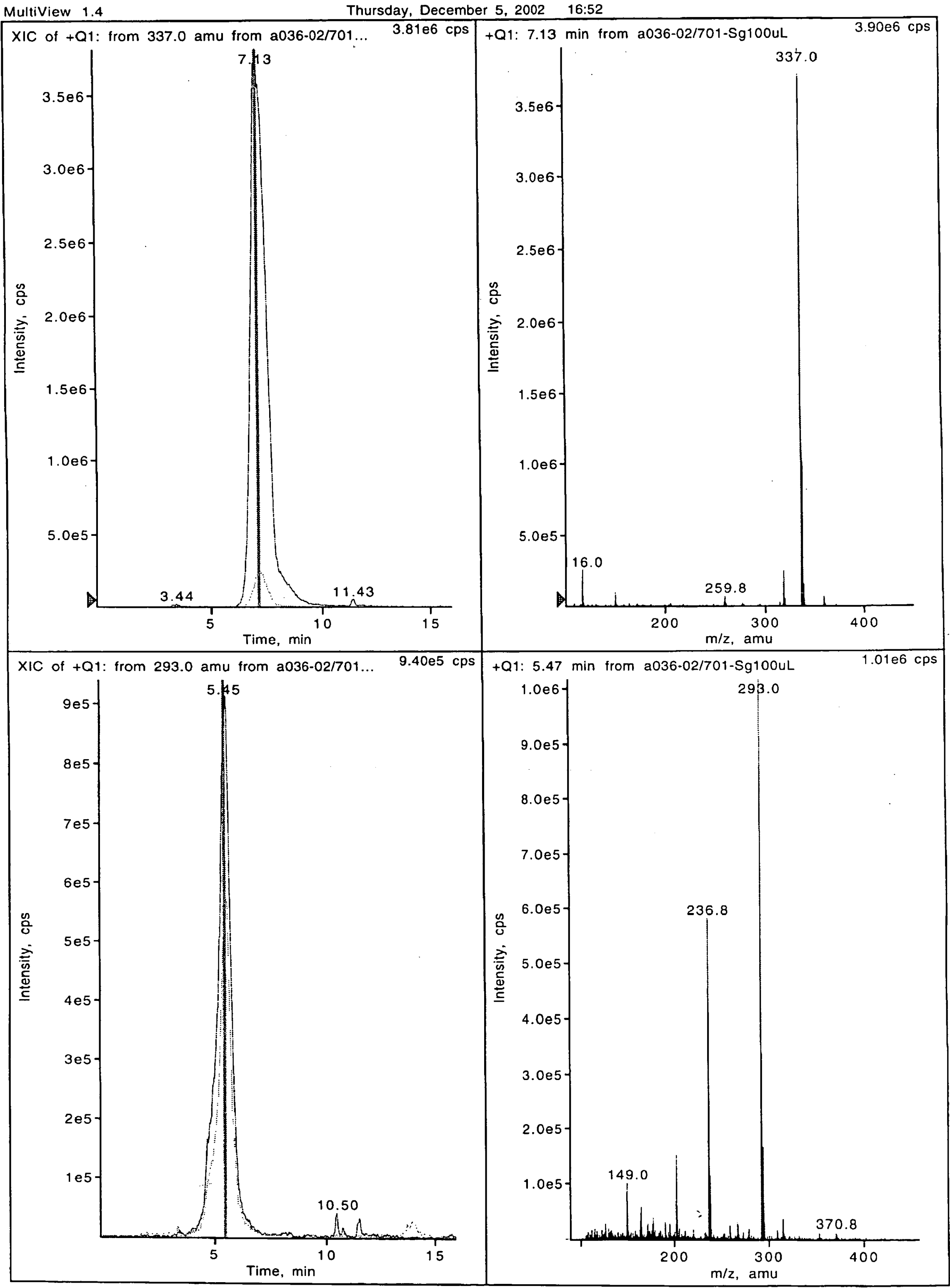

Figure 1 : Chromatographie de l'échantillon analysé par CLHP/SM pour la confirmation et la quantification de l'acébutolol. Partie du haut, à gauche : pic chromatographique de l'acébutolol, à droite : impact électronique. Partie du bas, à gauche : pic chromatographique du standard interne, à droite : impact électronique. 